\title{
Toward a New ICA Research Agenda
}

\author{
Liqiu Meng ${ }^{1 *}$, Temenoujka Bandrova ${ }^{2}$, Terje Midtbø ${ }^{3}$, Vít Voženílek ${ }^{4}$ \\ ${ }^{1}$ Chair of Cartography, Technical University of Munich,80333 Munich, Germany, liqiu.meng@tum.de \\ ${ }^{2}$ Department of Photogrammetry and Cartography, University of Architecture, Civil Engineering and Geodesy, 1046 \\ Sofia,Bulgaria, tbandrova@abv.bg \\ ${ }^{3}$ Department of Civil and Environmental Engineering, Norwegian University of Science and Technology, 7491 \\ Trondheim, Norway, terjem@ntnu.no \\ ${ }^{4}$ Department of Geoinformatics, Palacký University Olomouc, 77146 Olomouc, Czech Republic, vit.vozenilek@upol.cz \\ * Corresponding author: liqiu.meng@tum.de
}

Keywords: research agenda, ICA commissions, data science, cognitive science

\begin{abstract}
:
Since the publication of the first ICA Research Agenda of Cartography and GI Science in 2009 (Virrantaus et al. 2009), a lot has happened in terms of theoretical, methodological and technological developments related to the acquisition and management of big geospatial data, the design of cartographic products and map-based services, and the understanding of participatory map users. A working group on New Research Agenda of ICA was established by the Executive Committee of ICA after the ICC in Tokyo, 2019. It is dedicated to supporting the mission of ICA to advance the disciplines and professions of cartography and GIScience in an international context for the benefit of society and science. More specifically, the working group has a commitment to emphasize the role of research for ICA in creating, renewing and disseminating knowledge, and to maintain an up-to-date and overarching ICA research agenda as a living document.
\end{abstract}

The working group conducted in 2020 a survey with the aim to identify, on the one hand, the priority research topics of the individual ICA commissions and their preferred communication channels, and, on the other hand, the internal connections among ICA commissions as well as cross-disciplinary connections of ICA with other national, regional and international research communities. The results of the survey revealed dozens of individual research topics with varying frequencies and a number of contemporary cartographic research topic clusters as shown in Figure 1.

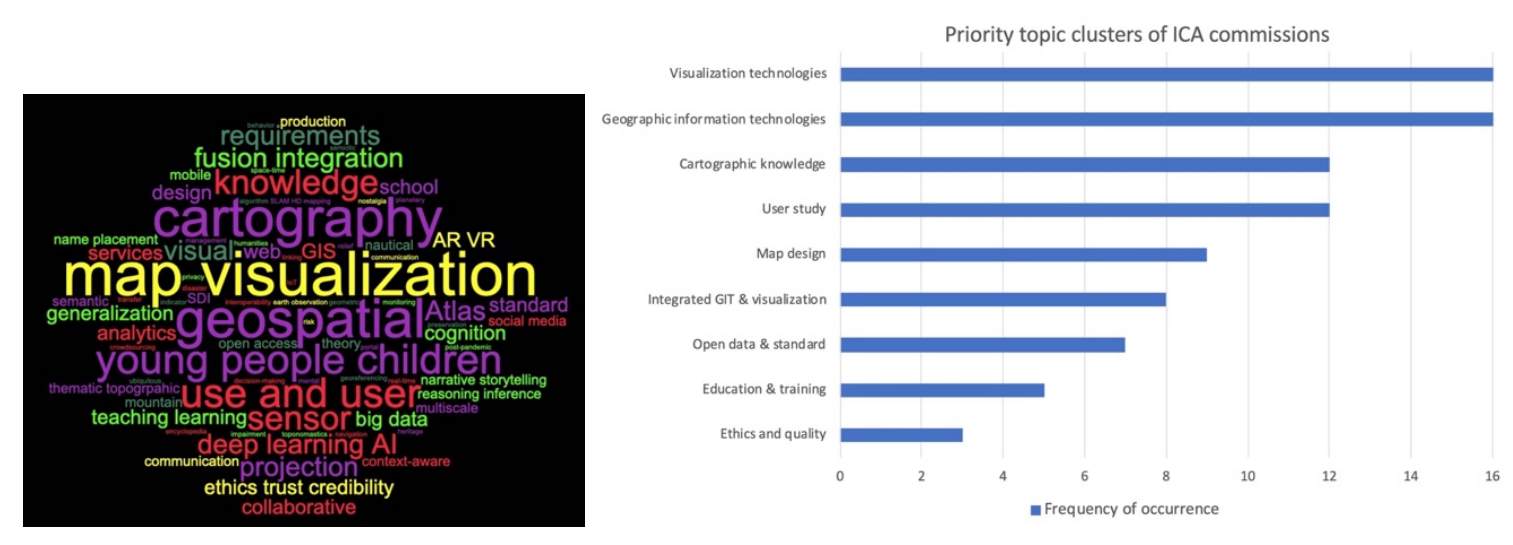

Figure 1. The occurrence frequency of individual research topics (left); Contemporary cartographic research topic clusters (right)

The working group is currently engaged in three new tasks:

- Revisit the previous ICA research agenda - The ten key phrases summarized in the previous ICA research agenda - (1) geographic information, (2) metadata and SDIs, (3) geospatial analysis and modelling, (4) usability, (5) geovisualization, visual analytics, (6) map production, (7) cartographic theory, (8) history of cartography and GI science, (9) education, and (10) society - are analyzed and compared with the aforementioned survey results. We aim to identify ad-hoc topics, emerging topics, long-standing topics, and the relative research intensity of these topics in different world regions. 
- Examine cartographic research highlights - By taking a close look at mission statements, research activities and publications issued by ICA commissions and worldwide cartographic research communities in recent years, we aim to identify best practice examples, analyze their driving forces, innovative ideas, methods, tools, and research findings, and assess their scientific value for enriching the body of knowledge for cartography, their transferability to other disciplines and their social impacts.

- Explore scientific references related to map, cartography and cartographer - Using automatic tools such as web crawlers, we collect and structure scientific references incl. journal publications, conference proceedings, technical reports, exhibition documents that indicate a sufficient relevance to map, cartography and cartographer. We then apply advanced analytical and machine learning tools to detect prominent topics and case studies that connect cartography with other disciplines in the global scientific landscape. Doing so will help us identify the need for interdisciplinary talent with cartographic skills, and prepare us to educate the next generation of cartographers and GIS scientists as "game changers".

In the long term, the working group will address a number of open questions triggered by recent developments of science, technology and humanity, such as:

- What is the role of cartography in Data Science and Citizen Science?

- What is the role of map in mobility research and autonomous driving?

- What kinds of business models for map production make sense for the sustainability of the cartographic discipline and profession?

- Does the claim that more than $80 \%$ of all information has some geospatial reference still hold true with the growing availability of social media data (Hahmann \& Burghardt 2013)?

- How can cartography and cognitive science (e.g., cognitive aspects of eye movements and brain activities, embodied cognition ) cross-fertilize in user studies?

- How can emotional factors be embedded in cartographic design principles, such as for map-based storytelling, journalistic map, to improve user experience?

- How are worldviews shaped by maps, e.g., Eurocentric maps?

- What ethical issues should be considered in cartography?

- What role do maps play in preserving the world's cultural heritage and sustainable development?

A number of preliminary findings about the evolution of cartography as an academic discipline in high education systems will be presented. These include the changing relationship of cartography to the geosciences on the one hand and the spatial cognition on the other. In addition, cartography is growing in many soil types across the Data Science and Open Science. It has an overarching responsibility for the quality and ethics of value chain of geodata. Cartography can help bring out the best in humans and AI.

\section{References}

Hahmann S and Burghardt D, 2013: How much information is geospatially referenced? Networks and cognition. International Journal of Geographical Information Science 27(6):1171-1189.

DOI:10.1080/13658816.2012.743664

Virrantaus K, Fairbairn D and Kraak M-J, 2009: ICA Research Agenda on Cartography and GI Science. The Cartographic Journal Vol. 46, No. 2, 63-75 